\title{
Automated Detection, Locking and Hitting a Fast Moving Aerial Object by Image Processing (Suitable For Guided Missile)
}

\author{
Atul Garg ${ }^{1}$, Redhawan Raziur Rouf ${ }^{2}$, Khandaker Nusaiba Hafiz ${ }^{3}$, Mehnaz \\ Sharna ${ }^{4}$, Nazmul Hasan ${ }^{5}$ \\ ${ }^{1}$ Professor (Military Institute of Science and Technology) \\ ${ }^{2,3,4,5}$ (Military Institute of Science and Technology)
}

\begin{abstract}
Due to passive tracking, fire and forget capabilities and ease of operation of guided missiles have emerged as an important anti-aircraft arsenal. Detection of target in real time is a difficult task when object is dynamic and even harder when the object tends to move at speed counted in Mach numbers. Moving target detection is a key area in image processing, infusion of this technology to the purpose of tracking target and destroying as in guided missiles can be very effective as it will reduce network links, and GPS navigation in aggressive electronic warfare environments. Detecting moving objects in real-time is a challenging problem due to the computational limits and the motions of the camera. In this paper, we suggest a method for dynamic object detection and tracking on non-stationary cameras running within few milliseconds (ms) on a PC, and real-time on mobile devices. Detecting of object by background subtraction method does not give better results when object is moving very fast, object is very tiny and presence of lighting effect. In order to overcome these troubles, we propose a new method for Moving object Detection in Dynamic Background along with the development of a guided missile using the process. It achieves dynamic landscape using certain possibility of time and subsequent frame difference method and addresses the difficult scenario, where object is moving very fast and background changes frequently. The experimental results show that the proposed method can detect moving object more efficiently and completely in both cases online as well as offline video. The results indicate that the proposed algorithm outperforms the majority of earlier state-of-the-art algorithms not only in terms of accuracy, but also in terms of processing speed.
\end{abstract}

Keywords: guided missile, image processing, new surface on missile- wing, artificial intelligence.

\section{Introduction}

Guided missile is an important element of air defence system. A number of methods are employed for guidance of an aerial weapon to its target. Every method has its merits and demerits. However, miss of a target by a misile can prove to very costly, due to any limitation. Image processing is one of the technology that can be employed for target detection and engagement. In this paper a method using image processing for target acquisition and guidance of a misile has been proposed. The method can enhance accuracy and performance of a misile.

\section{Missile Guidance}

A missile which is rocket propelled and whose flight path is controlled by radio signals or by internal preset or self-actuating homing devices during flight is called guided missile. This complete process is called missile guidance.

\section{Methods Of Missile Guidnce}

There are some technologies using to make a missile auto controlled. We will discuss about them to know these methods briefly.

Wire Guidance: It is an electromechanical system which controls vehicle steering by tracking an energized guide wire secured in the floor. The system is operated or sent it via thin wires connected between the missile and guidance mechanism.

Command Guidance: Command missiles are guided on the basis of electromagnetic radiation contact with a friendly source .It is a ground station or aircraft which relay signals to a guided missile via controlling radio and directed the missile where to steer in order to intercept the target.

Terrain Comparison Guidance: This is a navigation system which is primarily used by cruise missiles. It uses a pre-recorded outline map of the terrain which is compared to the dimensions made during flight by an 
on-board radar altimeter. This increases the precision of a missile compared to inertial navigation systems.

Terrestrial Guidance: It is a method of missile or rocket guidance in which the total flight path is controlled by making reference to the direction and strength of the earth's gravitational or magnetic field.

Inertial Guidance: It is an electronic system by which it monitors all the phase of a missile. It provides navigational data or control without need for communicating with a base station. Basic components which are used in an inertial guidance missile are gyroscopes, accelerometers and a computer. Fixed reference directions or turning rate measurements are provided by gyroscopes. Changing in the velocity of the system is measured by accelerometers. And lastly the computer gathers all the data and shows the final direction, velocity and makes a final result.

Beam Rider Guidance: The beam rider model depends on an outdoor ground or ship-based radar station that transmits a beam of radar energy in the direction of the target. The surface radar trails the target and also transmits a guidance beam that adjusts its angle as the target moves across the sky. It was mainly used in antiaircraft missiles and air defense systems.

Laser Guidance: In this system a laser beam is projected on the target, which is reflected from the target and hence gets scattered. The missile carries a laser seeker that can detect even infinitesimal amount of radiation. The receiver provides the direction of the laser scatter to the guidance system. The missile is launched in the direction of the target, the seeker searches for the laser reflections and the guidance system steers the missile towards the source of laser reflections i.e. to the target.

\section{Limitation Of Erlier Methods}

Presently, for missiles guidance, thermal guidance and laser guidance are most commonly used. But they have some limitation that may lead to guidance errors, resulting in probable target miss.

\section{Thermal guided missile:}

It is unable to recognize target body after descending to a high lower than $300 \mathrm{ft}$. These missiles can also be deflected by the use of flares.

\section{Laser guided missile:}

Since the missile tracks a laser signature, not the object itself, the target must be illuminated from a separate source, either from ground, by a pod on the attacking aircraft, or by a separate support aircraft.

\section{Image Processing}

Image processing is the method of detecting any body from a frame or video. Different methods are using for image processing. For our proposed method we will use background subtraction and frame difference method.

\section{Background Subtraction:}

For moving target detection background subtraction is frequently used. The basic scheme for subtracting background is to subtract the image of interest from a background image which is the reference image. The method has three steps. The first step is to construct a reference image froms a background image, while threshold selection is being done in the second step. Third step is pixel classification or subtraction of image. If the difference of pixels is greater than the threshold value, it considers it as a part of foreground and remaining part is consider as background.

\section{Frame difference:}

Frame difference (FD) is a basic background subtraction method. In this method the background and foreground can be detected very easily and in very short time [1]. In FD method we subtract the current frame from previous frame or from a reference frame to find out their difference to detect the foreground and background [2]. This method is commonly used to monitor objects on a video in medical, security, traffic management, communication, surveillances and other fields [3]. FD required very less memory to function. For detecting any object in FD method specific level of threshold $\mathrm{T}$ is used.

According to this scheme, pixels belongs to foreground if

$$
\begin{aligned}
& \left|I_{i}(x, y)-I_{i-1}(x ; y)\right| \quad T \ldots \ldots \ldots \ldots \ldots \ldots \ldots \ldots \ldots(1) \\
& R(x, y)=\left|I_{i}(x, y)-I_{i-1}(x ; y)\right| \ldots \ldots \ldots \ldots \ldots \ldots \ldots(2)
\end{aligned}
$$


$\boldsymbol{G}(\boldsymbol{x}, \boldsymbol{y})= \begin{cases}1 & \text { if } R(x, y)>T \\ 0 & \text { if } R(x, y) \leq T\end{cases}$

Where $\mathrm{I}_{\mathrm{i}}(\mathrm{x}, \mathrm{y})$ is the pixel for each point of current frame,

$\mathrm{I}_{\mathrm{i}-1}(\mathrm{x}, \mathrm{y})$ is the pixel for each point of previous frame and $\mathrm{T}$ is a chosen threshold suitable for that particular detection. When $G(x, y)$ is 1 , it is foreground and when $G(x, y)$ is 0 it is background.

This method is vulnerable and prone to errors for detection and speed of the object and rate of frame change. It is extremely sensitive to threshold value. It is also affected by noise and changes in illumination due to weather conditions. Hence it can detect all positions of the foreground [4] [5].

\section{Proposed Method}

The proposed method can be used for surface launched missiles. It will be piloted to the target area by manual controls with the help of GPS and radar system. The directional control of the missile will be carried out by the control surfaces.

On reaching the target area the missile will scan for the object and on acquiring the target, missile will switch over to auto mode. The target will be detected using the suggested image processing method. After the target detection and the identification will be confirmed by matching with the pre-fed pictures in its library. The flight controls will be exercised using artificial intelligence in the guided missile in response to the head movement of the camera mounted on the missile.

The motion of the camera head will provide the input to control system for missile directional control. The block diagram of the system will be as follows:

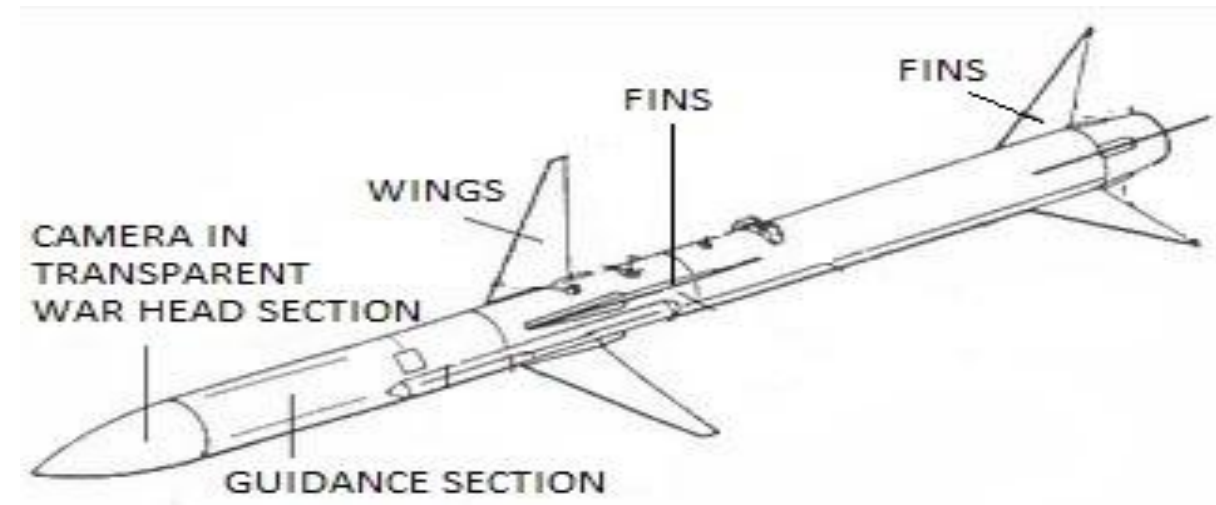

Fig 1: structure of a guided missile

The section bellow shows the attachment of the wing section to the guided missile. The wings are attached between processor and gyro.

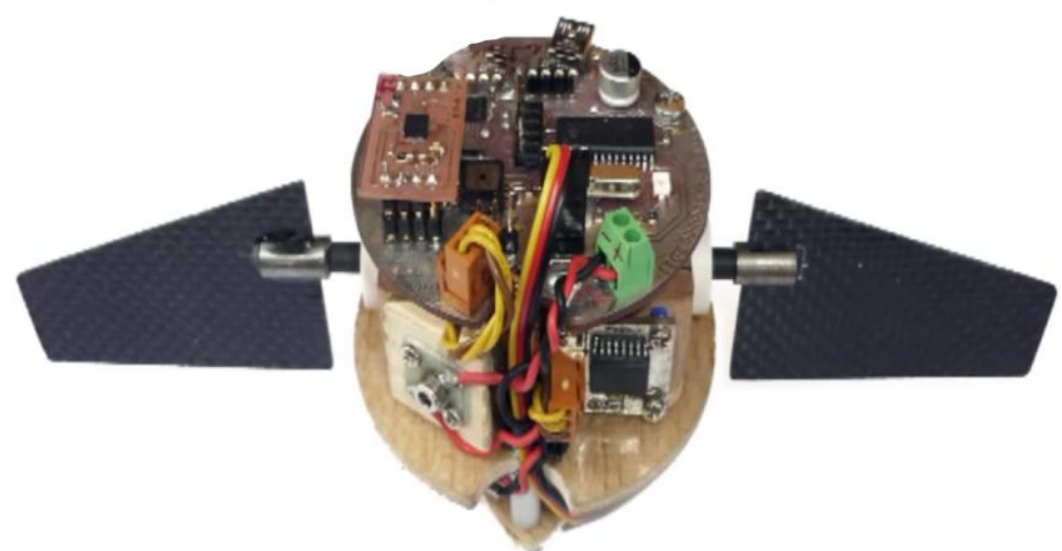

Fig 2: Image of the wing section with processor and gyro mounted 

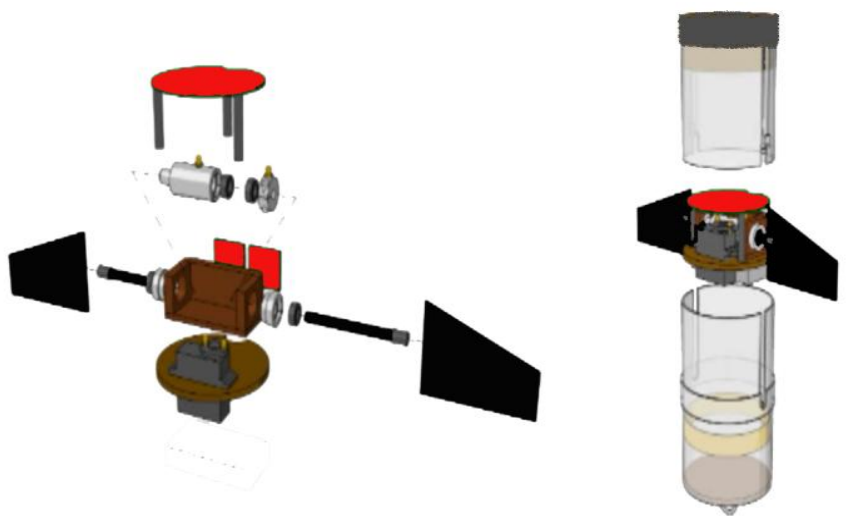

Fig 3: setting up of wings

The picture below shows the schematic diagram of the total setup of the proposed guided missile. The purple portion of the tail is propulsion section. Beneath the wings the red portion is the parachute which activates only if the guided system fails. The middle part is the wing section which has the processor and the gyro. The blue colored dome shaped portion houses the camera.

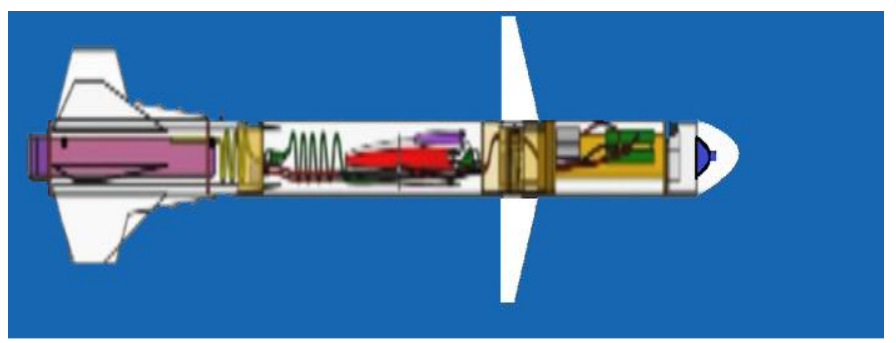

Fig 4: schematic model with wings in the center and camera in front inside warhead in blue

The cameras that will be used in this case must be gyro mounted to avoid the instability or bumpiness that will incurred during the motion of the missile. It is also must that the camera head must be able to rotate 360 degrees freely. A perfect example of the camera that can be used to serve such purpose can be as follows:

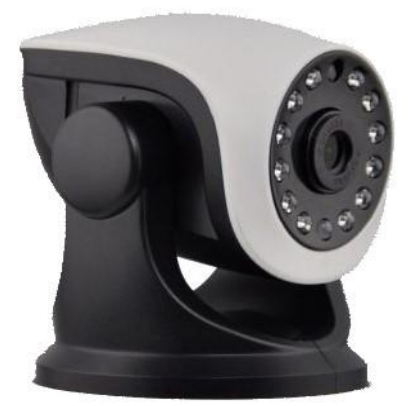

Fig 5: Example of the camera that can be used for the purpose

The 3D structure of the proposed guided missile was done in solid works and is given below:

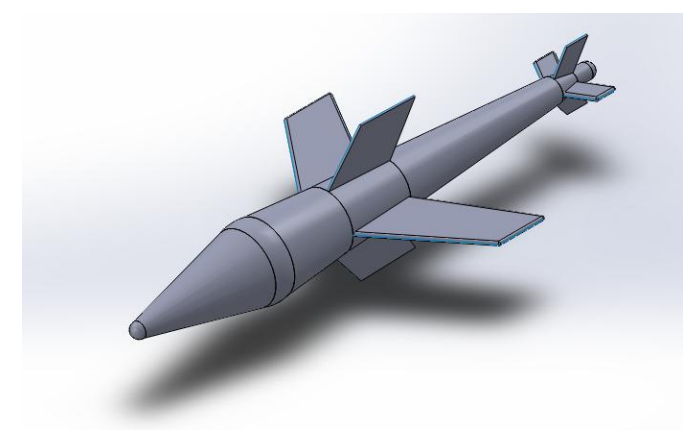




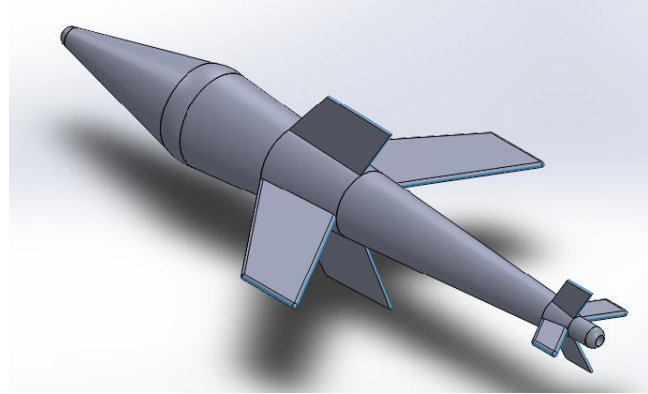

Fig 6: Solidworks design of the proposed guided missile

The simulation of the proposed missile system was done in CFD taking the speed of the body to be travelling at a speed of 8 Mach both in turbulent and laminar flow. The results are as follows:

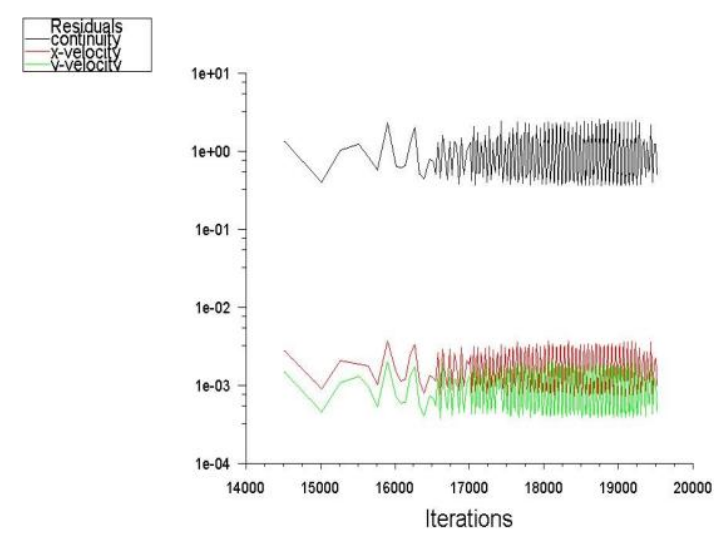

Fig 7: Scaled Residuals of the proposed guided missile in a speed of 8 Mach laminar flow

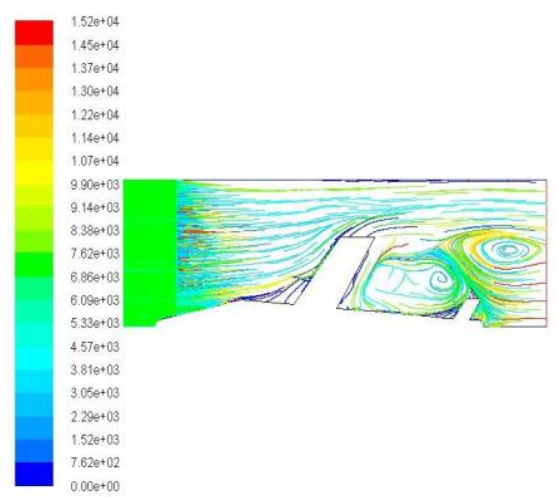

Fig 8: CFD simulation showing path lines colored by Particle ID in a speed of 8 Mach laminar flow

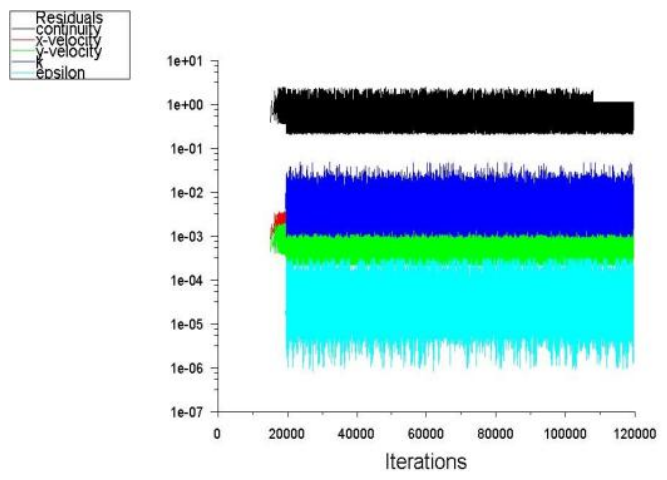

Fig 9: Scaled Residuals of the proposed guided missile in a speed of 8 Mach turbulent flow 


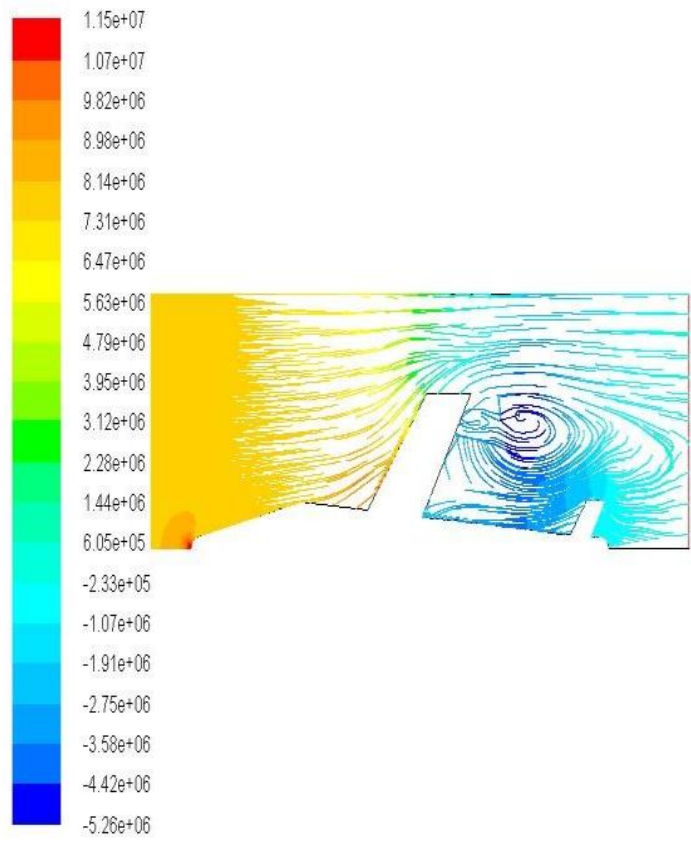

Fig 10: CFD simulation showing Path lines Colored by Static Pressure for the proposed guided missile in a speed of 8 Mach turbulent flow

\section{Detection Of Aerial Object By Image Processing}

In imaging science, image processing is processing of images using mathematical operations by using any form of signal processing for which the input is an image, a series of images, or a video, such as a photograph or video frame; the output of image processing may be either an image or a set of characteristics or parameters related to the image.

\section{A. Noise:}

Noise is any essence is not of advantage to the purpose of image processing. The effect of noises on the image signal amplitude and phase is not simplicity. So for smoothing the noise and keeping all the details of the image are the major tasks of image filtering.

\section{B. Noise filter:}

There are many methods of image filtering.Here we are using median method of filter.It's idea is not to use gray value of pixel point,it use ply the neighborhood pixel gray value.For the odd elements, it takes the middle value after sorting.For even elements ,the media takes the average size of the two middle values after sorting. It does not depend on the neighborhood with a lot of difference between typical values which can remove all types of impulse noise, salt, pepper noise frequently by retaining the image edge details. In general the median filters use odd numbered points of the sliding window.A first odd numbered pixel window is being determined by some specific methods. Each pixels in window line by the size of the gray value and use the location of the gray value between the image gray values as a substitute for enhanced values.

\section{Image segmentation:}

Image segmentation is a process in which the image is divided into some regions with similar characteristics and the target of interest is extracted.

\section{Threshold segmentation:}

Threshold segmentation has been used for image segmentation. The purpose is to identify the range of the gray scale threshold in the image and comparing all the image pixels gray values with the threshold. Based on the results, the corresponding pixel is divided into two categories, the foreground of or background.

There are two steps for threshold segmentation:

1. Determine the threshold T;

2. Compare threshold value with the pixel value.

Threshold value determination is one of the most critical steps. 


\section{E. Detection of the body:}

The detection of the body is achieved by image processing in frame differencing method as described earlier. The camera frame will have an amber colored box which will maintain the target within it. It will continue to follow the target until it correctly aligned with the missile. On having the target aligned the guided missile, it will create a red box on the target and generate hit command.

\section{Algorith Of Proposed Method}

Our proposed method will be shown by an algorith from the launching the missile to the hitting of target body.

1. The missle will be launched from a ground station and it will be manually control to bring the target body to missile's sight.

2. Missile will detect the target on first sight by image processing.

3. Missile command will be shift to auto control.

4. 4. It will check the position of the target body with the help of movement of the camera placed at the front of the missile.

5. Before hit the target body missile processor will check the target body's axis and the missile's axis are same or not to check if the target body is going to change the position.

This process will minimize the chance to miss the target body.

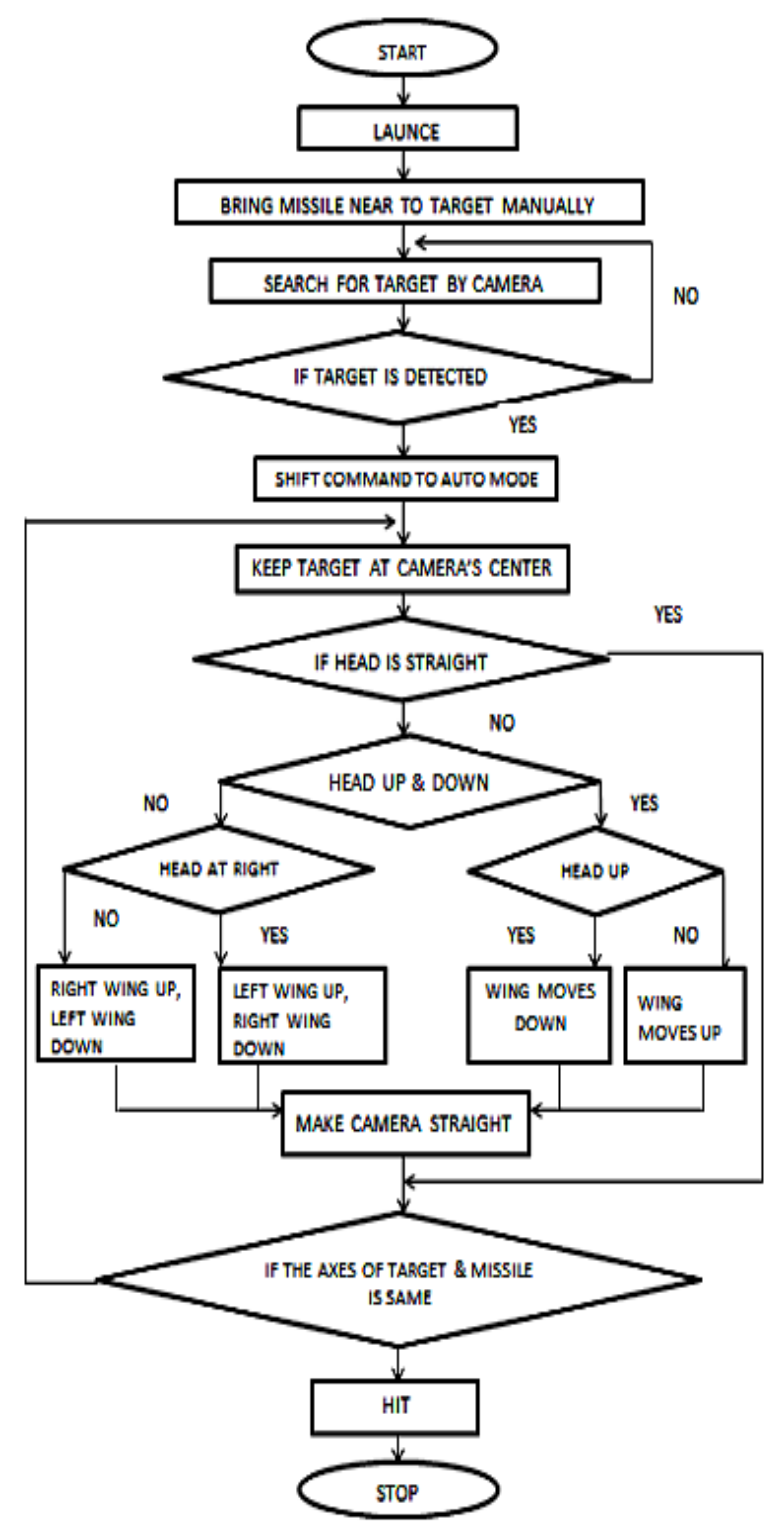

Fig 11: Algorithm 


\section{Simulation Result}

The simulation of the system proposed was carried out and the results were faster and precession was more accurate compared to other systems The different stage in the process of detection, locking and engaging of target object is given bellow:

STAGE 1: Input of the real time video to the processor via the camera mounted on the war head.

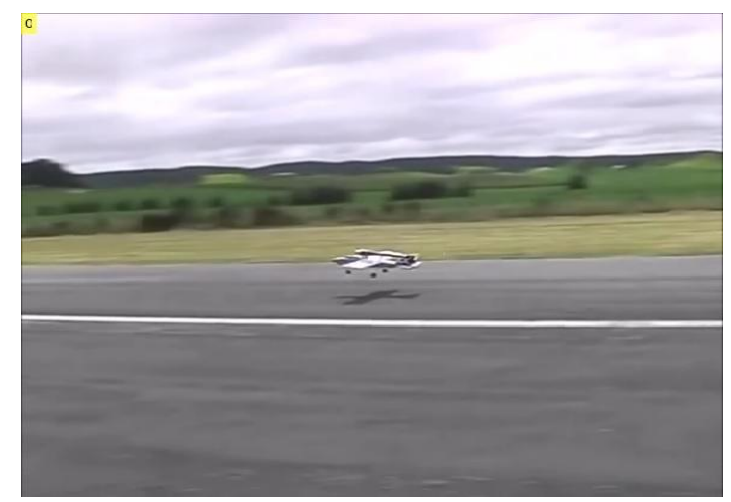

Fig 12: Projection of real time video

STAGE 2: Background subtraction of $t$ he input image and prediction of the location of the target object is determined by frame difference method. The predicted locations are spotted and bounded by a red rectangular box.

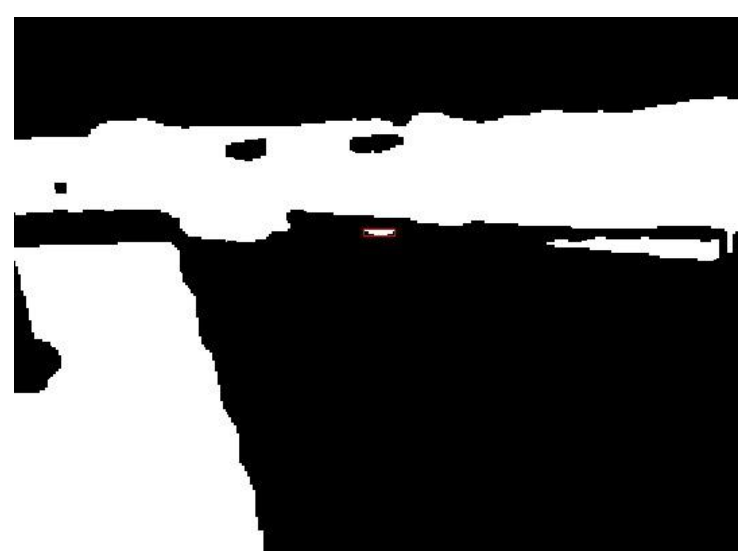

Fig 13: Background subtraction

STAGE 3: Matching of the detected object with the image pre-fed to the library of the system. When match is found the object gets locked by a rectangular box with green boundary line.

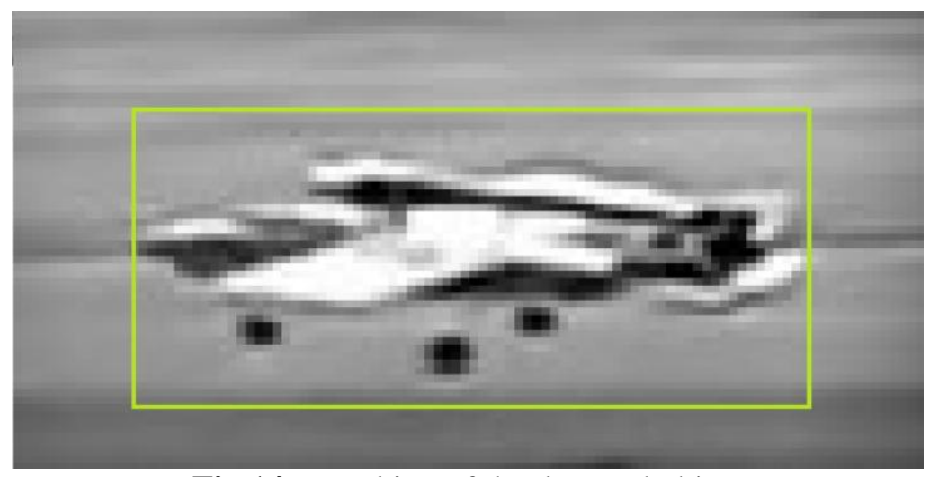

Fig 14: Matching of the detected object

STAGE 4: Locking of the target object is done in this stage and the camera tends to keep the object within the yellow colored rectangular box boy moving its head and hence shifting the direction of motion towards the targeted object. 


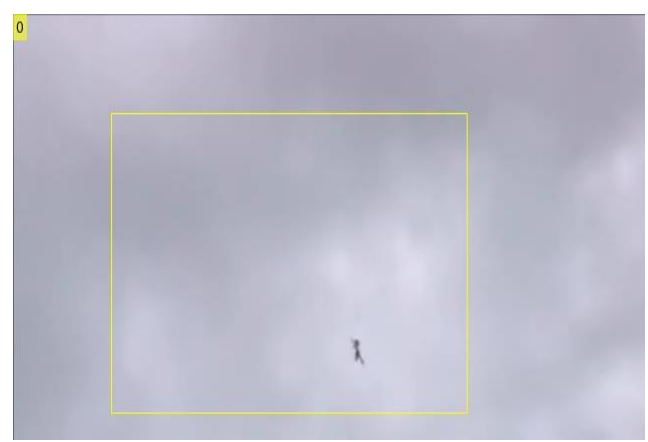

Fig 15: Oobject detection in target frame

STAGE 5: This stage commands the missile for final engagement of the target object. This occurs only when the Targeted object becomes aligned to the missile.

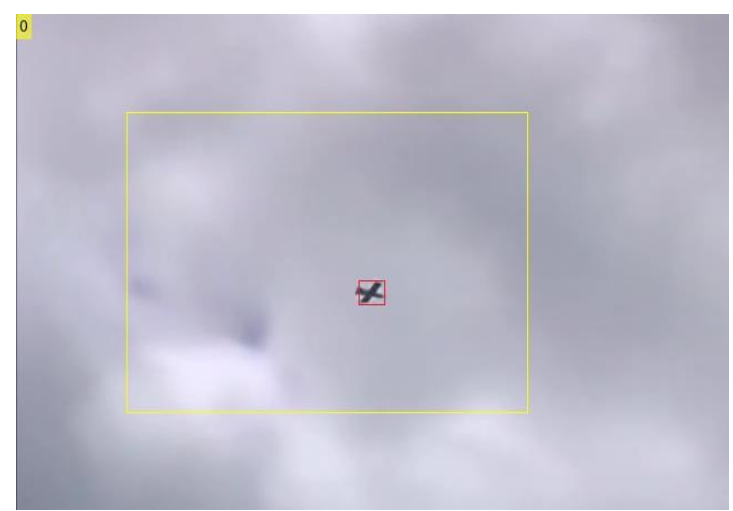

Fig 16: locking of target and ready to hit

\section{Conclusion}

The proposed system is more efficient in case of its purpose. Less time consuming and is more effective in cases where runways are unavailable and quick response is very much necessary. The advantages of our proposed method are-

1. As decision making remains within the missile so less time required for decision making.

2. Can perform maneuvers faster.

3. Cannot be distracted or no false body will be harmed as it matches its target body with the pictures predefined to its library.

The system will be further developed in case of its aerodynamic shape to help it move to supersonic speed and also developing the code to reduce time of processing.

\section{Reference}

[1]. Reid A. Shenq Wang Markandey, V. Motion estimation for moving target detection. IEEE Transactions on Aerospace and Electronic Systems, 32(3):886-874, 1996.

[2]. Nishu Singla," Motion Detection Based on Frame Difference Method", International Journal of Information \& Computation Technology, Volume 4, pp. 1559-1565, 15 Number 2014.

[3]. Wei Zeng, Wen Gao and Debin Zhao, "Automatic Moving Object Extraction in MPEG Video", IEEE Transaction on Signals and Image processing, pages: 524-527 2003.

[4]. A. Lipton, H. Fujiyoshi, and R. Patil,"Moving target classification and tracking from real-time video. Pages 8-14, Oct 1998

[5]. Yangquan Yu, Chunguang Zhou, Lan Huang, Zhezhou Yu, "A Moving Target Detection Algorithm Based on the Dynamic Background", Proceedings of the International Conference on Computational Intelligence and Software Engineering, pp.1-5, Dec. 2009. 